\title{
Urzidil wie Rothacker wie Watzlik? Johannes Urzidil als Grenzland-Dichter
}

Der Titel des Beitrages ist freilich mit Absicht provokativ gewählt, denn den Kennern der deutschböhmischen Literatur sind Autoren wie Rothacker und Watzlik, Strobl und Pleyer, Hohlbaum und Ott, Göth und Altrichter, Stauff und die Teichmann nur als anstößige Beispiele von Schriftstellern bekannt, die im ,Kampf ums deutsche Volkstum in Böhmen' an prominenten Stellen standen, die negativsten Auswüchse des Sudetendeutschtums repräsentierten, häufig die besonders abscheuliche Gattung des Grenzlandromans pflegten und nach 1945 die revanchistischen Ansätze eines Teils der Vertriebenen kanalisierten. Warum in aller Welt soll Johannes Urzidil, der letə̧te große Erzäbler der Prager Schule, der große Humanist, der Homo vere humanus, Prags Menscheitsdämmerer, der binternationale Troubadour des alten Prag - (so nur einige Überschriften von Aufsätzen über Johannes Urzidil) - warum soll Johannes Urzidil mit revanchistischen Autoren in einer Reihe genannt oder gar verglichen werden? Zumal seine negativen Stellungnahmen zu diesem Autorenkreis bekannt sind:

um in der Welt zu gelten, bedürfen die Prager deutschen Dichter keines einzigen nach 1900 im so genannten Sudetenland geborenen nichtjüdischen Dichters. Diese hingegen tun gut daran, sich an jene zu halten, wenn sie überhaupt irgendwo außerhalb ihrer eigenen Zirkel bemerkt werden wollen. (zit. n. Berger 1995: 243)

Es wäre freilich nur ein billiges Spiel mit Worten, wenn ich die Verbindung Urzidils zu diesen Grenzlanddichtern auf der bloßen Tatsache aufbauen würde, dass er eine seiner vielen böhmischen Erzählungen Grenそland nannte. Es wird zwar im Folgenden um diese Erzählung gehen, doch nicht nur um sie. Entstanden 1954, erstmals veröffentlicht $1955 \mathrm{im}$ Merkur, wurde sie in die erste, 1956 herausgegebene Erzählsammlung Urzidils, Die verlorene Geliebte, aufgenommen. Sie spielt - anders als die ihr vorangestellten Erzählungen (etwa Spiele und Tränen, Repetent Bäumel, Neujahrsrummel u. a.), nicht in Prag, sondern im Böhmerwald, hat - wiederum ähnlich wie die ihr vorangestellten Erzäh- 
lungen - einen autobiographischen Helden, ${ }^{1}$ der zugleich der Ich-Erzähler ist, und sie erzählt die Geschichte eines „merkwürdigen Kindes“ (Urzidil 1982: 129), der Stifter Otti. Otti Stifter, die ohne Mutter (womöglich eine weitere autobiographische Verbindungslinie zum Autor?), ${ }^{2}$ nur mit ihrem Vater, dem Stifter Anton, in der lieblichen und als Naturidylle dargestellten böhmerwäldischen Waldeinsamkeit lebt, verfügt über sonderbare Kräfte, die man geneigt ist, kurzerhand als magische Kräfte zu bezeichnen: Wenn sie den Arm ausstreckt, setzen sich Waldvögel drauf, wenn sie einen Stein aufhebt, beginnt er zu leuchten, wenn sie die Hand über Königskerzen bewegt, folgen diese ihrer Bewegung, wenn sie die Hand in den Bach taucht, schmiegen sich ihr liebkosend vorbeischwimmende Forellen an und die Wasserrosen bewegen sich auf sie zu. Sie sieht unter Moos verborgene Pilze, kann Regen und Gewitter, aber auch einen Brand im Dorf voraussagen, findet mit sicherem Griff verlegte Dinge, doch auch einen verlorenen Dorfjungen, zerbricht nie ein Ding, errät Stimmungen der Menschen, noch bevor diese sie selbst in sich wahrnehmen. Von der Außenwelt werden diese Fähigkeiten als „krank“, „abnormal“ und „verkehrt" (Urzidil 1982: 131f.) bezeichnet, weil sie sich jeglicher rationalen Deutung widersetzen. Auf die wiederholte Frage des Erzählers, der eine rationale Erklärung erzwingen will, „Wie machst du denn das? .... Wie stellst du das an?“ (Urzidil 1982: 129f.) antwortet Otti, „nur so“ (Urzidil 1982: 130) - und schon diese Erwiderung ist für sie außergewöhnlich rational (oder konventionell), denn sie benutzt die Sprache selten zur bloß konventionellen Kommunikation: Sie spricht selten ganze Sätze, sondern nur Satzfetzen, meistens nur deiktische Wörter, singt viel lieber, als sie spricht, singt den Dingen selbst erfundene Lieder in einer „völlig unverständlichen Sprache“, die „keinen Sinn ergeben $\cdot[. .$.$] sondern fast wie eine Zauberformel klingen." (Urzidil 1982:131) { }^{3}$ Auch als sie die Laune des Erzählers errät oder erzeugt, indem sie im Vorbeigehen fragt, „Weh?“ (Urzidil 1982: 130), ist ihr Sprachgebrauch magisch zu nennen, denn ihre Sprache beschreibt und bezeichnet nicht, sie schafft Wirklichkeit - was der Erzähler richtig deutet, indem er kommentiert:

1 Der Ich-Erzähler und die handelnde Ich-Figur werden zwar im sonderbaren Abstand voneinander gehalten - „In dieses unendliche Reich kam einmal einer, der hieß Ich.“ (Urzidil 1982: 126) - doch die autobiographischen Signale (die humanistische Bildung, das Schreiben als Beruf usw.) sind eindeutig.

2 Im späteren Verlauf der Interpretation wird sich allerdings zeigen, dass dieses Motiv nicht autobiographisch, sondern dem literarischen Vorbild entliehen ist.

$3 \mathrm{Ob}$ man deswegen Ottis Sprachgebrauch gleich als ,in die Zone des Zungenredens, der Glossolalie im frühen Christentum gerückt“ interpretieren muss (Trapp 2007: 44), bleibe dahingestellt. 
Ich hörte auf zu pfeifen und wusste nicht, ob ich traurig geworden war, weil sie das gesagt hatte, oder ob sie trotz meines Pfeifens erkannt hatte, dass ich traurig sei. (Urzidil 1982: 131)

Otti genießt freilich keine konventionelle Bildung durch Schule und Kirche, sondern zimmert sich ihre eigene Weltweisheit aus naturreligiösen, matriarchalisch gefärbten Vorstellungen. Dieses merkwürdige Kind zerbricht an der destruktiven Kraft des Eros (womöglich ein Nachklang der expressionistischen Phase Urzidils): ${ }^{4}$ Der bloße Anblick sich umarmender Liebender verwirrt sie, macht sie krank, beraubt sie ihrer Sicherheit im Umgang mit Dingen und Natur, beraubt sie ihrer magischen Kräfte. Der Erzähler erklärt sie für tot, noch bevor sie sich - nach dreimaliger verbaler Weigerung, zur Schulausbildung ins österreichische Kloster zu gehen - dem Druck der gesellschaftlichen Konvention, der Notwendigkeit, erwachsen und ergo ,wie die anderen“ (Urzidil 1982: 138) zu werden, endgültig durch den Suizid im See verweigert.

Diese Erzählung Johannes Urzidils hat - trotz des Titels - herzlich wenig damit zu tun, was man gemeinhin unter Grenzlandroman versteht, wie diese Gattung Michael Berger oder neuerdings Karsten Rinas charakterisierten:

Der grenzlanddeutsche Schriftsteller will in und mit seinen Werken den slawischen Verschlingungstrieb entlarven und das Erwachen des völkischen (eingeschlossen des rassischen) Lebenswillens eines Volkstums auf dem Weg von der Not- und Schicksalsgemeinschaft zur deutschen Volksgemeinschaft darstellen und befördern helfen. (Berger 1995: 265f.)

In der sudetendeutschen Grenzlandliteratur werden zeitgenössische Konflikte zwischen Deutschen und Tschechen thematisiert, wobei insbesondere um Sprache, Schule und die ökonomische Vorherrschaft gestritten wird. (Rinas 2008: 118)

Urzidils Grenzlanderzählung beinhaltet keines der genannten Motive, sondern knüpft die Verbindungslinien ganz woanders, bei Adalbert Stifter. Der große Böhmerwald-Dichter wird im Anfang der Erzählung explizit genannt, die Erzählung spielt in Stifterschen Kulissen, die Sprache der idyllisierenden Naturbeschreibungen ist die Sprache Stifters, sogar die Figur Ottis ist mit einer Stifterschen Figur nah verwandt, nämlich mit Ditha aus Stifters Novelle Abdias. Ich bin freilich nicht die erste, die auf diese Verbindung der beiden Texte aufmerksam macht, sondern es taten vor mir bereits Alfred Doppler (1986: 110f.), Johannes John (2000) und Gerhard Trapp (1967: 91-

4 Es gibt Interpreten, die - indem sie diese Erfahrung der weiblichen Heldin mit dem erotischen Erwachen eines männlichen Helden (des Hans in Morgen fahr ich heim aus Bist du es, Ronald?) vergleichen, dieses Motiv genderartig deuten, was $\mathrm{m}$. E. weder notwendig noch Urzidil angemessen ist (John 2000: 239). 
98; 2007), ${ }^{5}$ doch die Urheberschaft dieser Verbindung ist nicht so wichtig, wichtig ist, dass beide Figuren tatsächlich so nah verwandt sind, dass Urzidils bewusste Anleihe als ausgemacht gelten kann. Auch die mutterlose Ditha wird als ungewöhnlich bezeichnet. Ähnlich wie im Falle Ottis wird Dithas Andersartigkeit von der Außenwelt als Defizit wahrgenommen, sogar der liebende und umsorgende Vater „sah bald, dass das Mädchen nicht sei, wie es sein sollte““ (Stifter 1987: 321) und „Abdias konnte sich nicht helfen, er musste denken, dass Ditha blödsinnig sei.“ (Stifter 1987: 322) Der Erzähler Stifters bleibt bei der Bewertung der Andersartigkeit der Figur allerdings nicht in der Schwebe wie der Erzähler Urzidils, sondern findet einen rationalen - defizitären - Erklärungsgrund: Ditha ist blind geboren, was lange keiner sah. Mit Otti verbindet Ditha am stärksten ihr Sprachgebrauch: Sie spricht unbekannte Worte, ,die aus ihrem inneren Zustande genommen waren“, die niemand versteht, sie singt Lieder, die sie selbst erfindet, sie vermischt Sinneseindrücke in kühne Synästhesien ${ }^{6}$ und kommt den nicht verstehenden Anderen „etwa wie eine redende Blume“ (Stifter 1987: 341) vor. Ein weiteres Signal der StifterFiliation der Figur Ottis, bildet der Erklärungsversuch des „Amerikaners“ Feiferling hinsichtlich der magischen Kräfte Ottis: „Ich hab einmal etwas über ein magnetisches Mädel gelesen, die hat den Blitz angezogen“ (Urzidil 1982: 132), ein direkter Bezug zu Stifters Ditha, die sich als das äußerst gewittersensitives „magnetisches Mädel“ erweist, welches durch einen Blitz erst sehend und durch einen anderen Blitz schließlich getötet wird. ${ }^{7}$

Die Verbindungslinie der Erzählung mit dem sinnschweren und gewiss reflektierten und wohl überlegten Titel Grenzland (Urzidil hätte sie ja auch ganz anders nennen können: weder Stoff noch Inhalt ergeben notwendig den Titel, ${ }^{8}$

5 Den drei Interpreten geht es aber um die Stifter-Rezeption Urzidils, nicht um das Grenzland-Thema. Gerhard Trapp vergleicht Otti mit zwei weiteren weiblichen Gestalten der Literatur, Goethes Mignon und Stifters „braunem Mädchen“ aus der Erzählung Katæensilber.

6 „Als sie einmal [...] an einem großen blühenden Flachsfelde standen, rief sie aus: ,Vater, sieh nur, wie der ganze Himmel auf den Spitzen dieser grünen, stehenden Fäden klingt!' [...] So sprach sie auch von violetten Klängen und sagte, dass sie ihr lieber seien als die, welche aufrecht stehen und widerwärtig seien wie glühende Stäbe." (Stifter 1987: 341)

7 John (2000: 225) macht noch auf eine weitere Verbindungslinie aufmerksam, den Essay Urzidils über Abdias (Urzidil 1948).

8 Johannes John (2000: 238) deutet den Titel in Bezug auf die Grenzüberschreitung hin zum Erwachsenwerden nach dem erotischen Erlebnis Ottis, doch dafür ist die Erzählung - so scheint mir - zu wenig psychologisch, misst das ,Grenzland' des Pubertären nur sehr am Rande aus, diesem Motiv - trotzdem es freilich die Katastrophe einleitet - wird zu wenig Raum gewidmet, sodass es nicht plausibel schient, dass dadurch die Titelfundierung zu erklären wäre. 
den Raum, in welchem sie spielt, hätte man ja getrost auch ,Böhmerwald“ oder anders nennen können, zumal in der Erzählung nie explizit irgendeine Grenze genannt wird) zu Stifters Text, die - andererseits - vollkommene Unverwandtschaft dieser Erzählung mit den Vertretern der Granzlandromangattung sind so deutlich, dass man die These wagen könnte, dass Urzidil seine Erzählung bewusst gegen das geläufige Muster des Grenzlandromans geschrieben hat, dabei als Leitfolie die Gedankenwelt Stifters nutzend. Zur Unterstützung dieser These soll die in der Sammlung Die verlorene Geliebte nachfolgende Erzählung $W o$ das Tal endet herangezogen werden. Auch diese Geschichte begleitet den Ich-Erzähler in den Böhmerwald, auch in dieser Geschichte wird Stifter explizit erwähnt (der Ich-Erzähler liest Stifters Mappe meines Urgroßvaters und denkt über das „sanfte Gesetz“ nach), auch diese Geschichte hat eine merkwürdige Figur zum Haupthelden, den Dorftrottel Alois, ein Spiegelbild Ottis (im Gegensatz zu Otti zerbricht er - golemartig - alles, was ihm in die Hand kommt), seine Sprache ist noch reduzierter als die Ottis: Alois äußert sich nur durch „schrilles Gelächter oder winselndes Weinen“ (Urzidil 1982: 143), wobei aber beide Äußerungen, ,jeweils unter umgekehrten Vorzeichen erfolgen.“ (Urzidil 1982: 143) Wenn also den Ich-Erzähler bei seiner Ankunft ins Tal Alois”, wieherndes Lachen“ begrüßt, ist dies ein schlechtes Omen. Trotzdem folgt zunächst eine überaus witzige, ironisch-satirische Darstellung eines Dorfstreites zwischen den Rechts- und Linksbächlern in Hirschwalden, den Grünschmieden und Bierschimmlern um einen verschwundenen Quarkkuchen - die witzige Burleske endet allerdings mit einem Mord. Die Eskalation des Konflikts um triviale Ursachen, die einzelnen Schritte des lächerlichen Streites sind unschwer als Allegorie der tschechisch-deutschen Konfliktgemeinschaft in Böhmen zu dekodieren, obwohl der nationale Konflikt bis auf eine Ausnahme nicht explizit genannt wird. In diese Richtung weisen auch die eingestreuten Kommentare des Erzählers über die sich selbst potenzierende, sich verselbständigende, von den Ursachen abkoppelnde Eskalierung eines Konfliktes bzw. auch die Darstellung des Tales als Prototyp für Konflikte auf der ganzen Welt.

Unbeteiligt ist neben dem durchreisenden Erzähler lediglich der Förster Jelen, der nicht aus Hirschwalden, sondern aus dem Egerland stammt und die Herrschaft vertritt, welcher der Wald gehört und die von den Dorfbewohnern übereinstimmend gehasst und bestohlen wird. Jelen ist der einzige, der keine eindeutige nationale Zuordnung erfährt, dessen tschechisch-deutsche Ambivalenz betont wird: 
Er hieß Jelen, war aber ein Deutscher aus dem Egerlande, wobei zu bemerken wäre, dass der Name Jelen an sich tschechisch ist und ,Hirsch` bedeutet [...]. Die Familiennamen im zweisprachigen Böhmen waren zu allen Zeiten sehr untermischt. (Urzidil 1982: 142)

Jelen versucht im Streit zu vermitteln, wird jedoch als „Hergelaufener“, der sich lieber „um seine eigenen Angelegenheiten kümmern“ (Urzidil 1982: 148) solle, abgewiesen. Fehl schlagen auch die allerdings nur auf der Ebene der Erzählerreflexion erfolgenden Versuche des Erzählers, die zerstrittenen Dörfler auf die Stiftersche „Gemeinschaft des Waldes“ einzuschwören:

Immerhin schien es schon eine besondere Vergünstigung, mit dem Dichter des Waldes gerade in diesem Tal umzugehen, wenngleich sein sanftes Gesetz zu jener Zeit dort nicht waltete. (Urzidil 1982: 150)

Klar ist jedenfalls, dass alle drei am Konflikt unbeteiligten Figuren, ${ }^{9}$ die des Försters Jelen (dessen Namen man hier auch als einen typisch jüdischen $\mathrm{Na}$ men lesen könnte), ${ }^{10}$ die des - aus dem Abstand der inzwischen erfüllten Zukunft kommentierenden - Erzählers und die aus der Vergangenheit in die Gegenwart und Zukunft leitend hineinragende Figur Adalbert Stifters, für die andere Lösung des böhmischen Konfliktes stehen, die friedliche, die landespatriotische, die utraquistische. Diese andere Lösung scheitert jedoch an der Aggressivität des sich verselbständigenden Konflikts, doch vielmehr und viel endgültiger noch am Einbruch der großen Geschichte ins Hirschwaldener Theatrum mundi. Im Epilog der Erzählung fallen Allegorie und historische Realität in eins, werden zum apokalyptischen Symbol:

Die Frühlinge, die Sommer, die Herbste und die Winter zogen wohl darüber hinweg, aber über ihnen noch unheimlichere Wetterkatastrophen, die sich an keine natürliche Jahreszeit kehrten, apokalyptische Reiter und Sensemänner einer tieferen und gründlicheren Vernichtung. Und nach dieser kam von unten her eine neue Macht, die auch hier das Angestammte aus dem Boden riss und vertrieb. Sie achtete keiner Uferseite. Sie zwang am Ende allen das Reisebündel auf. [...] Das Tal hatte sich in Niemandsland verwandelt. (Urzidil 1982: 155)

9 Johannes John zählt zu den Unbeteiligten auch den Dorftrottel Alois, was aber eindeutig nicht stimmt, denn Alois beteiligt sich sehr wohl an Prügeleien unter den Kindern, beschleunigt den Gang des Konflikts durchs unerlaubte Melken der entwendeten Kuh, begleitet die Konflikthandlungen durch seine verkehrten Lach- und Weinäußerungen. Dafür zählt John Stifter nicht zum Ensemble der Unbeteiligten und Vermittelnden.

10 Mag sein, wie Gerhard Trapp (2007: 44) belegt, dass Jelen ein gleichnamiges reales nichtjüdisches Vorbild hatte, doch der Fluss meiner Argumentation berechtigt mich trotzdem dazu, in seinem Namen auch die Nennung der Gruppe der böhmischen Juden, die im tschechisch-deutschen Konflikt (wie bereits oft beschrieben) die Außensteher- und Außenseiterrolle hatten, mitzuhören. 
Dieser Epilog korrespondiert wiederum mit dem Prolog der Erzählung Gren₹land, wo es in den ersten Zeilen heißt:

In Oberplan und in den Dörfern des böhmischen Hochwalds gab es immer noch Leute, die Stifter hießen. [...] Selbst als die Gewalttätigkeit - verschiedenfarbig aber gleich widersinnig - sich dieser Landschaft bemächtigte, lebten dort noch Menschen mit dem Namen Stifter, bis auch sie davongehen mussten und sich dann irgendwo in der Fremde verloren. (Urzidil 1982: 125)

Die parabolische Botschaft der beiden Erzählungen ist hiermit überdeutlich, und die These, dass Urzidil - anders als seine böhmischen Generationsgenossen (die ihre Grenzlandromane und Grenzlanderzählungen allerdings mitten im tschechisch-deutschen Konflikt schrieben, nicht mit Abstand von fünfzehn Jahren, nach der globalen tragischen Beendigung und Auflösung des Konflikts) - eine andere Leitfigur, nämlich die Adalbert Stifters, und andere Leitgedanken in seiner Darstellung des Grenzlands benutzte und damit wahrscheinlich bewusst gegen die Gattung des Grenzlandromans anschrieb, wohl ebenfalls genügend bewiesen.

Bleibt die Frage, warum Johannes Urzidil, der sich nicht nur mit dem letzten Satz aus der Erzählung Wo das Tal endet als Dichter, als Anwalt der Vertriebenen zu verstehen gibt ${ }^{11}$ und der sich mit dem Titel Grenzland und der bewussten Orientierung des Grenzlandthemas auf Stifter und weg von der eingeübten Grenzlandgattung zum Grenzlanddiskurs meldete, warum Johannes Urzidil eben aus diesem Diskurs so gut wie hinausgefallen ist, äußerst selten als ,Grenzlanddichter ${ }^{6}$ wahrgenommen wird. Die Antwort ist wohl in der einführend zitierten Briefstelle zu erkennen, in der Urzidil selbst eine scharfe Trennlinie zwischen der Prager deutschen und der sudetendeutschen Literatur zieht: Etwa vom Anfang der 60er Jahre an ist die Forschung um die deutschböhmische Literatur darauf bedacht, eine Grenze zwischen den ,zwei deutschsprachigen Literaturen' aus Böhmen und Mähren zu ziehen, zwischen der Prager deutschen und der, anderen', wobei die , andere Literatur' im Verhältnis zur Prager deutschen eine ästhetische und kulturpolitische Abwertung erfuhr, die ihr bis heute anhaftet. ${ }^{12}$ Diese Abgrenzung mag zwar

11 „Und ich gehöre noch zu den letzten, die das irrsinnige Gelächter über diesem See der Gewalt von ferne her vernehmen und seinen warnenden Sinn verstehen." (Urzidil 1982: 155) Urzidils eindeutige Verurteilung der Vertreibung der Deutschen aus Böhmen ist ebenfalls in seinem Stifter-Essay Der Blick vom Stingelfelsen (1965; auch in Bekenntnisse eines Pedanten, 1972) nachzulesen.

12 Einflussreich sind in diesem Zusammenhang nach wie vor die Formulierungen des tschechischen Germanisten Eduard Goldstückers (1967: 21, 24f.), der in Anknüpfung an seine Definition der Prager deutschen Literatur auch die sudentendeutsche Literatur erwähnt: 
die ästhetische, intellektuelle und politische Profilierung der Prager deutschen Literatur in den 20er und 30er Jahren kennzeichnen und auf zeitgenössische Texte von Max Brod, Pavel Eisner, Ludwig Winder, Otto Pick sowie auf spätere Memoiren der Prager Autoren (wie die Johannes Urzidils) zurückgehen. Nichtsdestoweniger etabliert sie ein exkludierendes und normatives Schema mit groben Vereinfachungen und einer stereotypen Wahrnehmung. Die deutsche Literatur aus der Provinz wird an der ästhetisch und auch ethisch höherstehenden Prager deutschen Literatur gemessen und vereinfachend mit der stigmatisierenden Bezeichnung sudetendeutsche Literatur belegt und abqualifiziert, deren Kennzeichen neben revanchistischem Nationalismus, Antisemitismus und biederer Heimatpflege auch ein triviales Kunstverständnis sei. Die so definierte sudetendeutsche Literatur wird dann von der Prager deutschen Literatur abgegrenzt, eine Vermengung der beiden Diskurse - des allein der sudetendeutschen Literatur zugeschriebenen Grenzland- und Vertriebenendiskurses und der der Prager deutschen Literatur - ist so gut wie undenkbar. Kein Wunder, dass selten ein Interpret sich wagte, beide zu verschränken, Johannes Urzidil - den ,letzten großen Erzähler der Prager Schule ${ }^{\text {(13 }}$ - in die Nähe, selbst wenn es eine kontradiktorische Nähe sein sollte, der sudetendeutschen Grenzlanddichter Pleyer, Watzlik, Strobl und anderer zu rücken. ${ }^{14}$

Es gab in den böhmischen Ländern „,noch eine andere, in deutscher Sprache geschriebene Literatur, die sich als ,deutschböhmisch' bezeichnete. Dieser Ausdruck wurde nach dem ersten Weltkrieg und insbesondere nach der nationalsozialistischen Machtergreifung durch die Bezeichnung ,sudetendeutsch' ersetzt. Es handelt sich im wesentlichen um eine regionale Literatur, deren Verfasser meistens auf dem Standpunkt eines militanten deutschen Nationalismus [...] standen. [...] Die Ausnahmen betreffen einmal die sozialistische [...], zum anderen die Literatur, die zwischen den zwei Kriegen den Weg zu einem vernünftigen Zusammenleben von Tschechen und Deutschen [...] suchte. Als ihre markanteste Persönlichkeit muss zweifellos Josef Mühlberger angesehen werden. [...] Die Prager deutsche Literatur in unserem Sinn unterscheidet sich von dieser so genannten sudetendeutschen Literatur dadurch, dass kein einziger ihrer Verfasser [...] den militanten nationalen Standpunkt gegenüber den Tschechen einnahm und selbstverständlich keiner von ihnen unter dem Einfluss des Antisemitismus stand. Diese, sudetendeutsche' Literatur [machte] zu ihrer Zeit viel böses Blut [und ist heute] so gut wie spurlos verschwunden.“

13 So Ernst Schönwieses (1980) Schule machende Bezeichnung für Johannes Urzidil.

14 Die Betrachtung der - von Klaus Johann zusammengestellten - Bibliographie der Sekundärliteratur ergab, dass von den 721 Einträgen (mittlerweile umfaßt sie 804 Einträge, s. $<$ http://www.urzidil.de>) sich bloß ca. 15 auf den sudetendeutschen Kontext beziehen lassen, wobei ich diesen Bezug auf zweierlei Wegen herstellte: durch die Nennung eines sudetendeutschen Themas/Autors im Aufsatz (wie etwa Alena Kovarikova: Hans Watzlike und Johannes Urzidil. Zwei Heimatbilder - etwa die Hälfte der 15 Bibliographieeinträge) oder durch die Publikation eines Textes über Johannes Urzidil im Publikationsorgan, das traditi- 
Ich wollte mit meinem Beitrag Johannes Urzidil, sein Werk, seine Gedankenwelt mitnichten in das Niemandsland zwischen der Prager deutschen und der sudetendeutschen Literatur rücken und Urzidil, „den entschiedenen Gegner der Eingliederung des Sudetenlandes ins deutsche Reich" John 2000: 224) des Kontextes der Prager deutschen Literatur berauben, den er ja mit seinen Nachkriegs-Werken kräftig mitgeholfen hat zu gestalten, sogar ins Mythische zu überhöhen, sondern wollte nur zeigen, dass ein Aufbrechen ideologisch diktierter Diskursgrenzen den analysierten Text hin und wieder erhellen mag.

\section{Literatur}

Berger, Michael (1995): Von der böhmischen Heimat ins sudetendeutsche Grenzland. Differenzierungsprozesse in der deutschböhmischen Literatur von 1848 bis 1939. - In: brücken NF 3, 241-277.

Doppler, Alfred (1986): Wie sieht Johannes Urzidil Adalbert Stifter? - In: Lachinger, Johann/Schiffkorn, Aldemar [sen.]/Zettl, Walter (Hgg.), Johannes Urzidil und der Prager Kreis. Vorträge des römischen Johannes-Urzidil-Symposions 1984. Linz: AdalbertStifter-Institut des Landes Oberösterreich, 107-112.

Goldstücker, Eduard (1967): Die Prager deutsche Literatur als historisches Phänomen. - In: Ders. (Hg.), Weltfreunde. Konferenz über die Prager deutsche Literatur. Praha: Akademia, 21-45.

John, Johannes (2000): Adalbert Stifter in Erzählungen von Johannes Urzidil. - In: Hettche, Walter/Ders./ Steinsdorff, Sibylle v. (Hgg.), Stifter-Studien. Ein Festgeschenk. für Wolfgang Frübwald zu seinem 65. Geburtstag. Tübingen: Niemeyer, 217-243.

Rinas, Karsten (2008): Die andere Grenzlandliteratur. Zu einigen tschechischen Romanen mit antideutscher Tendenz. - In: brücken NF 16, 115-163.

onsgemäß dieser Szene zugeordnet wird (wie etwa Sudetenland). Der zweite Bezugsweg sagt aber eigentlich weniger über Urzidils Beziehung zum Sudetendeutschtum aus als vielmehr über die Beziehung der Vertriebenenkreise zu Urzidil und deren Versuche, Urzidil als den ihrigen zu vereinnahmen. - Eine genauere Untersuchung dieser Beziehung steht noch aus, genauso wie die Untersuchung der Frage, inwieweit bzw. wann und wann nicht mehr sich Urzidil vereinnahmen ließ. 
Schönwiese, Ernst (1980): Literatur in Wien zwischen 1930 und 1980. Wien, München: Amalthea.

Stifter, Adalbert (1987): Eræählungen. Stuttgart: Parkland.

Trapp, Gerhard (1967): Die Prosa Johannes Urzidils. Zum Verständnis eines literarischen Werdegangs vom Expressionismus zur Gegenwart. Bern: Lang.

Trapp, Gerhard (2007): Aspekte der Stifter-Rezeption bei Johannes Urzidil. - In: Jahrbuch des Adalbert-Stifter-Institutes des Landes Oberösterreich 14, 41-48

Urzidil, Johannes (1948): Adalbert Stifter and Judaism. - In: The Menorah Journal 36/4, 327-338.

Urzidil, Johannes (1982): Die verlorene Geliebte. Nachwort v. Oskar Holl. Frankfurt/M., Berlin, Wien: Ullstein. 